\title{
Franz Berto
}

\section{Adding 4.0241 to TLP}

\begin{abstract}
Tractatus 4.024 inspired the dominant semantics of our time: truthconditional semantics. This is focused on possible worlds: the content of $p$ is the set of worlds where $p$ is true. It has become increasingly clear that such an account is, at best, defective: we need an "independent factor in meaning, constrained but not determined by truth-conditions" (Yablo 2014: 2), because sentences can be differently true at the same possible worlds. I suggest a missing comment which, had it been included in the Tractatus, would have helped semantics get this right from the start. This is my 4.0241: "Knowing what is the case if a sentence is true is knowing its ways of being true": knowing a sentence's truth possibilities and what we now call its topic, or subject matter. I show that the famous "fundamental thought" that "the 'logical constants' do not represent" (4.0312) can be understood in terms of ways-based views of meaning. Such views also help with puzzling claims like 5.122: "If $p$ follows from $q$, the sense of ' $p$ ' is contained in that of ' $q$ ', , which are compatible with a conception of entailment combining truth-preservation with the preservation of topicality, or of ways of being true.
\end{abstract}

The way history has unrolled, the old containment idea was beaten out by the notion of truth preservation.

- David Kaplan, The Meaning of "Ouch" and "Oops"

\section{How Did We Get From There to Here?}

We've all heard it so many times:

4.024 To understand a proposition means to know what is the case, if it is true.

Franz Berto, University of St Andrews, UK, and University of Amsterdam, NL, fb96@standrews.ac.uk 
Tractatus Logico-Philosophicus (TLP)'s section ${ }^{1} 4.024$ has been at the core of the dominant semantics of 20th Century: truth-conditional semantics. This has focused, as a matter of historical fact, on truth-at-possible-worlds conditions. The meaning or content of a sentence is given by its intension: a function from possible worlds to truth values or, equivalently, the set of possible worlds where the sentence is true. Call this Standard Possible Worlds Semantics (SPWS).

Possible worlds don't show up explicitly in the TLP. Let's thus start by laying on the table some textbook Tractarian semantics - by which I mean: a minimal account, which doesn't get into big interpretative issues. ${ }^{2}$ Say that an atomic sentence is one that doesn't have further sentences as its syntactic constituents. Such a sentence is meaningful by being a picture of a state of affairs (4.032): a possible configuration or combination of objects, perhaps of objects and properties/relations $(2.01,2.031-2.032,2.202)$. The state of affairs is the sense of the sentence qua picture $(2.221,3.13)$, what we know by understanding it (4.021). States of affairs can obtain, or fail to obtain. Obtaining states of affairs are facts (The TLP's terminology is not very uniform here, but let's not quibble over this either). By obtaining, a state of affairs makes true the sentence it is the sense of (4.25). The world is the totality of facts, that is, of obtaining states of affairs (1-1.2, 2.04-2.06, 2.063). Thus, all the true atomic sentences taken together make for a complete description of the world (4.26). Complex sentences are truth-functional compounds of atomic sentences (5): their senses consist of the combinations of the obtaining and non-obtaining of the states of affairs pictured by their atomic constituents, which make them true (4.2, 4.4-4.41, 4.431).

How did we get from (something like) this to SPWS? Carnap is usually taken as the main responsible. While introducing his method of intension and extension in Meaning and Necessity, he claims that "some ideas of Wittgenstein were the starting-point for the development of this method" (Carnap 1947: 9). Notoriously, he talked of state descriptions, not possible worlds, but a state description is "a class of sentences [...] which contains for every atomic sentence either this sentence

1 I will call "sections" the numbered sentences, or groups thereof, composing the TLP (TLP); these are sometimes called "propositions", but I find the terminology a bit confusing. I will use "sentence" as short for "declarative sentence": a linguistic configuration that can be true or false. The TLP uses "Satz" for this, and the Ogden translation has "proposition"; I will only leave the word with this meaning when directly quoting the TLP. I myself will use "proposition" or "(propositional) content" for the meaning or content of a declarative sentence.

2 Like, What exactly is an elementary or atomic sentence? Does it include predicative terms? What's an atomic fact? How do the objects that constitute an atomic fact hang together? Are properties and relations objects, too? What are Tractarian objects like, by the way? - and so on. There are many excellent guides to the TLP, giving overviews of the debates on these issues. I recommend especially Frascolla 2007 and White 2006. 
or its negation, but not both, and no other sentences" (Ibid.). Ignoring various complications, one may take them as close enough to complete descriptions of possible worlds - or, as the worlds themselves, in a linguistic ersatz (Lewis 1986, Ch. 3) account of them (Carnap claims, a bit misleadingly, that "the state-descriptions represent Leibniz' possible worlds or Wittgenstein's possible states of affairs”, Ibid). Equivalently, one can take the totality of possible states of affairs, and form maximally consistent combinations of them. These will be, in a combinatorial setting, the possible worlds. Let their collection be $W$. The actual world is but the totality of states of affairs that obtain. And then we have it: the intension of a sentence $p$, giving its propositional content (or so-called UCLA proposition), is a function $I_{p}: W \rightarrow\{T, F\}$ mapping each $w \in W$ to its extension: truth, if $p$ is true at $w$; falsity otherwise.

\section{Aboutness, Topics, Subject Matters}

The last decades have made increasingly clear that the SPWS account of propositional content is, at best, defective. According to Yablo, we need an "independent factor in meaning, constrained but not determined by truth-conditions" (Yablo 2014: 2). That's because sentences can be differently true - true in different ways - at the same possible worlds, and SPWS tells us very little on how and why sentences are true at the relevant worlds. Take for instance:

Equilateral triangles are equiangular.

$2+2=4$.

These are true at the same worlds (all of them, if mathematical necessity is unrestricted) but they don't seem to mean or say the same thing: only one is about triangles, and made true by how they are.

One may quibble over the use of necessary truths, but take an example made famous by Hempel:

All ravens are black.

All non-black things are non-ravens.

These are (classically) logically equivalent, thus true at the same worlds, and arguably contingent, but they cannot quite mean the same if, as it seems intuitive, different pieces of evidence confirm them. Only one is about ravens, and made true by how ravens happen to be. 
According to Yablo, aboutness is the missing ingredient in SPWS. This is "the relation that meaningful items bear to whatever it is that they are on or of or that they address or concern" (Yablo 2014: 1): their subject matter or, as I shall also call it, their topic. I'll say something on what topics or subject matters are, or could be, in a minute. Here's what they are not - or, cannot easily reduce to: the things referred to in a sentence. Topicality also has to do with what is said about those things. "Dog bites man" and "Man bites dog" (Yablo 2014: 34) involve the same things: dog, man, perhaps biting - but they don't say the same.

The main alternative to SPWS for an account of propositional content comes from structured propositions. Can't they get it right? In the so-called Russellian view, the content of "John kisses Mary" is a structured object involving John, Mary, and the relation of kissing, in some order. Ordering can tell it apart from the content of "Mary kisses John". There are several problems with structured propositions as an account of content and same-saying at the right level of semantic fine-grainedness. I cannot get into them here but, for an insightful discussion, one can read Ripley 2012. I'll just mention one issue, raised in Yablo 2014: 1, for it will be important in the following: in the Russellian account, "Mary does not kiss John” differs in content from "Mary kisses John", in that the content of the former includes a component, not, which the content of the latter lacks. Also, the content of "Mary kisses John and Paul is jealous" includes and as a component. This seems wrong for subject matters: there's no sense in which "Mary does not kiss John" talks about negation, or "Mary kisses John and Paul is jealous" talks about conjunction.

Work on subject matter and ways of being true has been flourishing lately. This has revitalized a semantic tradition to some extent alternative to SPWS, and focused on a mereological view of meaning, whereby contents can be taken as having parts, as including other contents, as capable of being fused into wholes which inherit the proper features from the parts. Ideas concerning subject matters can be found already in Parry 1933, Ryle 1933, Goodman 1961, Perry 1989. Ways of being true have more recently been understood in terms of partial content (Humberstone 2000), world-partitions (Lewis 1988), world-divisions (Yablo 2014, Osorio-K. 2016), truthmakers (Fine 2014, Fine 2015), and more (Gioulatou 2016, Hawke 2017).

I don't think that the semantic ideas included in the TLP lead inevitably to SPWS. I'm not a specialist of Wittgenstein - only an admirer of the TLP since I was an undergrad. Still, it seems to me that there are, in it, traces of an alternative, mereological view of content. Before proposing, in two sections, a "missing gloss" to TLP 4.024 which, had it been there, would have helped 20th Century semantics get things right from the start, in the next section I will dig into a place in the TLP where such traces seem to show up: the Tractarian account of logical consequence. 


\section{The Uncomfortable 5.122}

SPWS can easily make sense of 5.12 and its first subsection:

5.12 In particular the truth of a proposition $p$ follows from that of a proposition $q$, if all the truth-grounds of the second are truth-grounds of the first.

5.121 The truth-grounds of $q$ are contained in those of $p: p$ follows from $q$.

The truth-grounds of a sentence are "those truth-possibilities of its truth-arguments, which verify the proposition" (5.101). These can be understood via the notion of Tractarian logical space (1.13, 2.11, 2.202, 3.4, 3.42, 4.463), whose mainstream interpretation is in terms of possible worlds (Stenius 1960) and relies, essentially, on the Carnapian ideas sketched above: each possible world is a combination of the obtaining and non-obtaining of states of affairs depicted by the atomic sentences. Then 5.12-entailment is close enough to truth preservation at all worlds in all interpretations or models, as per SPWS.

The problems come with the second gloss to 5.12:

5.122 If $p$ follows from $q$, the sense of " $p$ " is contained in that of " $q$ ".

Here the direction of containment is the other way around: the conclusion is included in the premise. A related idea pops up in 5.14: "If a proposition [ $p$ ] follows from another $[q]$, then the latter $[q]$ says more than the former $[p]$, the former $[p]$ less than the latter $[q]$ ". The entailing $q$ says at least whatever the entailed $p$ said, thus including it in some sense. As pointed out in the beautiful Negro 2017: 5, it is not straightforward to understand this in terms of SPWS. Negro notices that 5.122-consequence has given headaches to several authoritative interpreters of the Tractatus. Here's Ramsey:

I think this statement is really a definition of containing as regards senses, and an extension of the meaning of "assert" partly in conformity with ordinary usage, which probably agrees as regards $p \wedge q$ and $p[\ldots]$ but not otherwise. (Ramsey 1923: 471, notation adapted for consistency)

Ramsey's way of making sense of sense containment has it that, when by asserting $p$ we happen to assert $q$, the sense of $p$ has that of $q$ as a part. As an intuitive ("conforming to ordinary usage") example, he points at the sense of " $p \wedge q$ "s including that of $p$. Because $p \wedge q$ entails $q$ according to SPWS, this does not tell sense containment apart from SPWS logical consequence. But there's another intuitive example in the vicinity, which does: $p$ entails $p \vee q$ according to SPWS, but surely " $p \vee q$ " can be partly about something $p$ is not about, namely whatever $q$ is about. We'll get back to both entailments later. 
Max Black is more outspoken than Ramsey: he calls the idea of sense containment "an impediment to clarity", and glosses on 5.122 by speaking of "a peculiar (and possibly unfortunate) use of this word [scil. 'contained']” (Black 1964: 251 252). Fogelin 1976 says that sense containment is no more than a metaphor.

Can we make sense of sense-containment? Negro 2017 and Frascolla 2007 have interesting proposals (differing from each other in ways I won't get into here), which pivot on looking, rather than at truth-grounds, at their complements, namely, falsity-grounds, in a negative or exclusionary view of content (Rumfitt 2008). We can then explain the reversed inclusion direction of sense containment as some kind of inclusion of falsity-grounds.

Now I want to leave Wittgensteinian exegesis behind, though, and broaden the view a bit. There is a less-than-metaphorical tradition in logic and semantics variously stretching back at least to Kantian ideas on analyticity - of looking for content-preserving entailment relations: relations that hold between $p$ and $q$ only when $q$ introduces no content alien to that of $p$. Among such relations are those labeled as "tautological entailment" (Van Fraassen 1969), "analytic containment" (Angell 1977), “analytic implication” (Parry 1933, Fine 1986, Ferguson 2014). I will sketch two ways to approach content-containment and ways-based semantics in the coming section, after I've added my proposed gloss to 4.024.

\section{4.0241*, and Two Ways to Ways}

Here's my main proposed addition to TLP, meant to help semantics move beyond SPWS (let me flag it with an asterisk, to clarify that it's my stuff, not Wittgenstein's):

$4.0241^{\star}$ Knowing what is the case if a sentence is true is knowing its ways of being true.

How do we understand such ways of being true? One way to have a ways-based account of propositional content is found in Kit Fine's works, e.g., Fine 2014, Fine 2015. Fine's core idea is very Tractarian in spirit: sentences are made true by states of affairs or situations, rather than by whole possible worlds. His semantics has a set with a partial ordering on it, $\langle S, \leq\rangle$. Each $s \in S$ is a situation, or state of affairs, or a configuration of objects, or of objects and properties. States of affairs are things that can obtain or fail, and are chunks of reality that ground the truth and falsity of sentences, so we're not tremendously far from TLP's own states of affairs.

We are far from possible worlds, however - though one can recover a certain account of worlds by taking them as limit constructions out of states. The ordering $s_{1} \leq s_{2}$ may be read in a metaphysically loaded way: " $s_{1}$ is a real part of $s_{2}$ ", thus, 
states of affairs can be literally included in larger ones (this works well mainly with states of affairs involving concrete entities: the state consisting of St Andrews' being in Scotland is included in the state consisting of St Andrews' being in the UK). I favor a less metaphysically loaded reading of " $\leq$ " in terms of information: " $s_{2}$ preserves all the information in $s_{1}$ ", or " $s_{2}$ supports the truth of anything whose truth is supported by $s_{1}$ ", or so. Then what matters about states of affairs, for semantics, is the information they encode or support.

Either way, worlds would be things which are maximal with respect to $\leq$ : when $w_{1}$ and $w_{2}$ are worlds, the only way for " $w_{1} \leq w_{2}$ " to hold is for $w_{1}$ to be $w_{2}$. Finean states are not like that: like the situations of Barwise/Perry 1983's situation semantics, they are partial and relevant for the things whose truth they support. And it's states, not worlds, which are at the core of Kit Fine's semantics - just as they are at the core of the Tractarian theory of representation: it's of facts, i.e., (obtaining) states of affairs, that we make pictures of (2.1). Pictures are facts (2.141): the facts consisting in their elements' being in such-and-such relations with one another (2.14). The elements of the picture stand for (vertreten) the objects which are the constituents of the pictured state of affairs (2.131) - and so on, as per the well-known (though variously interpreted) pictorial theory of representation. Thoughts are logical pictures, (3), and sentences are the expression of thoughts, (3.1). The sense of a sentence - its content - is the state of affairs pictured by the sentence. What I understand when I grasp the content of $p$, is the state of affairs $p$ represents (4.021).

I will not get into the details of Fine's state-based semantics, however, for I favor a different way of having a way-based semantics. This comes from two-component (2C) accounts of content, which are more friendly to worlds. One account of this kind has been developed in great detail in Yablo 2014, but I'll stick to a more abstract 2C setting I'm working on, together with some friends (Berto 2018a, Berto 2018b, Hawke 2017). The content of a sentence $p$ is the thick proposition it expresses, [[p]]. This has two components: (1) $p$ 's intension, $|p| \subseteq W$, its truth set as per SPWS (the thin UCLA proposition), and (2) $p$ 's topic or subject matter, $t(p)$. Overall, $[[p]]=\langle|p|, t(p)\rangle \cdot p$ and $q$ say the same, that is, express the same thick proposition, when (1) they are true at the same worlds, and (2) they have the same topic. Given that topics can have parts, we also get a natural view of content inclusion: the content of $p$ includes that of $q$ when (i) any $p$-world is a $q$-world and (ii) the topic of $q$ is part of that of $p$. When both (i) and (ii) obtain, we claim that $p$ "says more than (or, at least as much as)' $q$.

Two natural questions now are, Why two components? What are these topics or subject matters? As for the first one, our understanding of propositional contents can be naturally seen as involving two elements. Now I attempt a further envisaged 
addition to the TLP - a subsection glossing on my proposed $4.0241^{\star}$, and pushing more in the $2 \mathrm{C}$ direction:

4.02411* One knows how a sentence is true by knowing its truth possibilities and what it is about.

One understands what $p$ means or says, when one understands (1) what the sentence speaks about, its topic or subject matter, and (2) what it says about that topic or subject matter. One understands "La neige est blanche" as soon as one knows that (1) it speaks about the color of snow, how snow is like, snow's whiteness, etc. - and, one knows that (2) it says that things are such-and-so with respect to that subject matter, that is, that snow is white.

In a plain state-based account à la Fine, the two components are taken care of by one kind of things: the states. A state of affairs verifying $p$ is both what $p$ is about, and what truthmakes it. But we may have tentative reasons for splitting the two components. It seems that people can sometimes have a partial understanding of the meaning of a sentence by grasping only its truth conditions, or only its topic. William III could have understood the truth conditions of "Either France will get into a nuclear war with England, or not”: he'd know, by only looking at its logical form, that the sentence would be true no matter what. He couldn't have grasped what the sentence is about, for he had no idea of what a nuclear war is. William III could have grasped the topic of "Louis XIV is bald" (say, Louis' baldness) without knowing under which conditions the sentence would be true (just in case Louis had $n$ hairs - for what $n$ ? A range between some $n$ and some $m$ ? Which $n$ and $m$ then? A fuzzy range? etc.).

That we split components doesn't mean that we must treat them as conceptually or metaphysically irreducible to each other. Here our second question comes in: What are topics, or subject matters? One may take them, again, as truthmakers or states of affairs, and reduce worlds to them by taking worlds as maximally informative states, or constructions out of states. But one may also go the other way around. This was the way pursued in Lewis 1988's seminal work on subject matters. Understand subject matters starting from questions: the subject matter of $p$ (in context $c$ ), is given via the question or questions $p$ can be taken as answering to (in $c$ ). This determines a partition of the set of worlds: $w_{1}$ and $w_{2}$ end up in the same cell when they agree on the answer. "The number of stars is eight" has as its topic the number of stars. It can be taken as an answer to the question, How

3 I won't get in detail into the following issue in this paper, but: as subject matters are contextdependent, it makes sense to say that the same sentence can get different subject matters in different contexts insofar as it answers to different questions: "Matteo Plebani is a communist' can be paired to, What are Matteo's political preferences?, or to Who are the communists in the 
many stars are there? This splits and groups the worlds depending on how they answer: all the zero-star worlds end up in one cell; all the one-star worlds end up in another; etc.

Yablo 2014 proposes to generalize Lewisian partitions, thus equivalence relations, to divisions: sets of worlds that collectively cover the modal space, but which allow overlap, determined by similarity relations which are reflexive and symmetric, but not transitive. Some questions have more than one correct answer ("Where can I find a B\&B in Kirchberg?"), so a world can be in more than one cell with respect to them. In any case, subject matters reduce to ways of splitting and grouping worlds into sets.

Before I give you more 2C details, I need to talk of Wittgenstein's fundamental thought.

\section{$54.0312,2 C$, and Back to 5.122}

Whether one takes a Fine-style, a 2C-style, or some other approach, a key principle followed in accounts of subject matter goes hand in hand with what Wittgenstein took as his "fundamental thought" in the TLP: that the logical constants represent nothing, for "the logic of the facts cannot be represented" (4.0312).

In a motto: the logical vocabulary contributes nothing to topicality, or subject matter. Let's stick with the Boolean connectives. ${ }^{4}$ We saw that there is something wrong in the claim that "Snow is not white" is about negation. "Snow is not white" must be about whatever "Snow is white" is about: that may be snow's color, the whiteness of snow, etc. In general, “ $\neg p$ ” must be exactly about what $p$ is about. We can phrase this first guiding principle for a propositional recursion on topics in 2C-terms: $t(\neg p)=t(p)$. Thus, also, $t(\neg \neg p)=t(p)$. Remember Frege on the Sinn-preservation of double negation, a view endorsed by Wittgenstein:

4.0621 That, however, the signs " $p$ "and " $q$ " can say the same thing is important, for it shows that the sign “ " corresponds to nothing in reality.

That negation occurs in a proposition, is no characteristic of its sense $(\sim \sim p=p)$. [...]

$5.44[. .$.$] And if there was an object called “ ", then " p" would have to say something other$ than " $p$ ". For the one proposition would then treat of $\sim$, the other would not.

Plebani family? - etc. A nice development of subject matter theory in this direction is Plebani/Spolaore 2018.

4 The view could be readily extended to the universal and particular quantifiers, if one took them as generalized conjunctions and disjunctions, respectively; but there are some subtleties involved here I don't want to get into. 
As for conjunction and disjunction: " $p \wedge q$ " and “ $p \vee q$ " have the same subject matter: a fusion or merging of the subject matter of $p$ and that of $q$ : "John is tall and handsome" and "John is tall or handsome" are both about the same topic, namely the height and looks of John's. (Again, that doesn't make them express the same thick proposition: their truth sets are distinct.) Where “ $\oplus$ ” stands for topic fusion, the second guiding principle for a propositional recursion on topics is: $t(p \wedge q)=t(p \vee q)=t(p) \oplus t(q)$. Again, the logical vocabulary is topic-transparent.

We can now build a small $2 \mathrm{C}$ semantics. Let's have a plain propositional language $\mathcal{L}$ with atoms $\mathcal{L}_{\mathcal{A T}}: p, q, r\left(p_{1}, p_{2}, \ldots\right)$, negation $\neg$, conjunction $\wedge$, disjunction $\vee$, round parentheses (), as auxiliary symbols. We use $A, B, C\left(A_{1}, A_{2}, \ldots\right)$ as metavariables for formulas. The well-formed formulas are the atoms and, if $A$ and $B$ are formulas: $|\neg A|(A \wedge B)|(A \vee B)|$.

A model for $\mathcal{L}$ is a tuple $\mathfrak{M}=\langle W, T, \oplus, v, t\rangle$, where:

- $W$ is a nonempty set of possible worlds;

- $T$ is a nonempty set of possible topics;

- $\oplus$ is an idempotent, commutative, associative binary operation on $T$;

- $v: \mathcal{L}_{\mathcal{A T}} \rightarrow \mathcal{P}(W)$ assigns a truth set $v(p)=|p|$ to each atom $p$;

- $t: \mathcal{L}_{\mathcal{A T}} \rightarrow T$ assigns a topic $t(p)$ to each atom $p$.

Out of fusion we can define what it means that topic $x$ is part of topic $y, x \leq y=d f$ $x \oplus y=y$ - making of parthood a partial ordering (with the strict ordering, $<$, defined from the nonstrict $\leq$, the usual way). $v$ is extended to the whole $\mathcal{L}$ via the usual recursive clauses assigning a truth set $|A|$ to each formula $A$ of $\mathcal{L}$ :

$-|\neg A|=W-|A|$

- $|A \wedge B|=|A| \cap|B|$

- $|A \vee B|=|A| \cup|B|$

We also extend $t$ to the whole $\mathcal{L}$ following our two guiding principles above:

$-t(\neg A)=t(A)$

- $t(A \wedge B)=t(A \vee B)=t(A) \oplus t(B)$

This double recursion gives us a fully recursive assignment, to each formula $A$ of $\mathcal{L}$, of a thick propositional content $[[A]]=\langle|A|, t(A)\rangle . A$ and $B$ say the same, i.e., they express the same content (in model $\mathfrak{M})$, when $[[A]]=[[B]]$, that is, $|A|=|B|$ and $t(A)=t(B)$ (and, one easily defines "saying at least as much as" and "saying strictly more"). 
This account of propositional content is strictly more fine-grained than what one gets in SPWS, given that any difference in truth set will make for a difference in thick proposition, but there will be additional distinctions warranted by different topic-assignments, e.g., $[[\neg(A \wedge \neg A)]] \neq[[B \vee \neg B]]$ ("It's not the case that the Liar is both true and untrue” doesn't say the same as "Either Goldbach's Conjecture holds, or not"). Still, lots of sentences turn out to express the same thick proposition, e.g., $[[A \wedge B]]=[[B \wedge A]]$ ("It's rainy and cold today" and "It's cold and rainy today" say

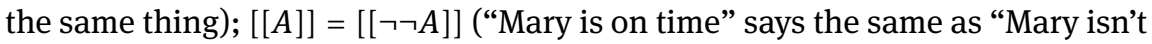
late”); [[ $A \vee \neg B]]=[[\neg(A \wedge B)]]$ (“Either Mary doesn't like John or she doesn't like Paul” says the same as "Mary doesn't like both John and Paul”).

Now back to Wittgenstein. We saw that, as pointed out by Negro, one cannot straightforwardly understand sense containment, as per 5.122, merely in terms of SPWS truth sets. But one can understand it by taking propositional contents as more than truth sets. 2C-semantics can get close enough to the spirit of 5.122 via a characterization of logical consequence (say, $A \vDash B$ : “ $B$ follows from $A$ ", or " $A$ entails $B$ "), that embeds topic-containment. We now know what it means, in a 2C-setting, that one thick proposition includes another: $[[A]]$ includes $[[B]]$ (let's write “[[A]] $\unrhd[[B]]$ ”, that is, $\langle|A|, t(A)\rangle \unrhd\langle|B|, t(B)\rangle)$ when (1) $|A| \subseteq|B|$, all the $A$-worlds are $B$-worlds, and (2) $t(B) \leq t(A)$, that is, the topic or subject matter of $B$ is included in that of $A$. We can interpret in 2C terms "A proposition affirms every proposition that follows from it" (5.124), and "If one proposition follows from another, then the latter says more than the former, and the former says less than the latter" (5.14): we have precise characterizations of same-saying, saying strictly more, saying strictly less, saying at least as much. In particular, we claim that $A \vDash \mathfrak{M} B$ just in case $[[A]] \unrhd[[B]]$ in $\mathfrak{M}$, that is, $A$ says at least as much as $B$ there: while $A$ 's truth set involves no more worlds, its subject matter is at least as big as that of $B$. And we say that $A \vDash B$ when $A \vDash \mathfrak{M} B$ for all $\mathfrak{M}$.

\section{Conclusion: Pulling in Different Directions}

I wouldn't want to claim that all of the TLP can be made consistent with a topicsensitive account of propositional content, whether of the $2 \mathrm{C}$ kind, or of some other sort - and in particular, that 4.0241*, and especially 4.02411*, don't betray the TLP in any way. On the contrary, the book often pulls in an opposite direction. One of the many things clarified by Negro 2017, is that Wittgenstein himself admitted, some years after the publication of the TLP, that his ideas around sense containment were less than fully clear when he wrote the book. In his Notebook I, which dates back to the late Twenties or early Thirties, Wittgenstein says: 
If the proposition $q$ follows from the proposition $p$, then I thought that $p \wedge \neg q$ has to be a contradiction and this I saw quite rightly. I believed that I had to further infer from this [...] that $q$ in some sense has to be contained in $p$. For if both propositions had nothing to do with each other, how could $p \wedge \neg q$ be a contradiction? In what sense $q$ is supposed to be contained in $p$ does not yet emerge from this [...] and I did not clearly specify it [...]. There is a clear sense in saying: $q$ is contained in the logical product $p \wedge q$. (Baker 2003: 127 and 197, notation adjusted for consistency.)

Perhaps Wittgenstein had clear the obvious case of content containment given by $A \wedge B$ 's entailing $A$, but couldn't readily see how to generalize it to a full-fledged notion of content-preserving entailment: since "he had only intuitively grasped the general case as correct, [this] was thus left completely unexplained in the Tractatus" (Negro 2017: 6).

In the TLP, $A$ entails $A \vee B$ in the 5.12 sense of entailment: all the truth-grounds of the former are truth-grounds of the latter or, as we would say today, any possible world in any interpretation or model making $A$ true will also make $A \vee B$ true. But, unlike $A \wedge B \vDash A$, this entailment is not topic-preserving, for $B$ may break the boundaries of $A$ 's subject matter: it is not the case that, in general, whatever " $A \vee B$ " says was already said by $A$. These have been taken as paradigmatic cases of content inclusion and noninclusion, and as data a theory of content-preserving entailment must comply with:

A paradigm of inclusion, I take it, is the relation that simple conjunctions bear to their conjuncts - the relation Snow is white and expensive bears, for example, to Snow is white. A paradigm of noninclusion is the relation disjuncts bear to disjunctions; Snow is white does not have Snow is white or expensive as a part. (Yablo 2014:11)

A guiding principle behind the understanding of partial content is that the content of $A$ and $B$ should each be part of the content of $A \wedge B$ but that the content of $A \vee B$ should not in general be part of the content of either $A$ or $B$. (Fine 2015: 1)

Here the hiatus comes to the fore:

4.465 The logical product of a tautology and a proposition says the same thing as the proposition. Therefore that product is identical with the proposition.

Thus, in particular, $A$ says the same as $A \wedge(B \vee \neg B)$. If one only looks at truth sets, these coincide. But it needn't be the case that $t(A)=t(A \wedge(B \vee \neg B))$, so the two cannot express the same thick proposition. In fact, I think that the interpretation of TLP sense-containment provided by Negro in terms of inclusion of falsity-grounds (Negro 2017: 17 - 20) does a better job than my little 2C semantics in making sense of the whole stance of the TLP with regards to logical consequence and content inclusion. 
However, when it's about characterizing propositional content and samesaying, it's the thick proposition view that gets it right, not the merely truthconditional view - or so I claim. How can "Jane fell out of bed" say the same as "Jane fell out of bed and either John is 6 feet tall or not"? Only the latter is, partly, about John's heights. Mary may have brought it about that Jane fell out of bed without bringing it about that Jane fell out of bed and either John is 6 feet tall or not (Perry 1989).

It still seems to me reasonable to say, at least, that the direction into which truth-conditional semantics has de facto been developed into SPWS and UCLA thin propositions is not straightforwardly mandated by the TLP. One should not be puzzled by the puzzlement of authoritative interpreters like Ramsey or Black: of course they had a hard time coordinating what the TLP says about sense containment in 5.122 with the direction it takes elsewhere. I'm more puzzled by the fact that the authoritative interpreters already saw the merely truth-conditional direction as the default one, and treated the other one, the content-containment direction, as the one which shouldn't have been there, or should have been treated as a mere metaphor. ${ }^{5}$

\section{Bibliography}

Angell, R.B. (1977): “Three Systems of First Degree Entailment.” In: Journal of Symbolic Logic. Vol. 47, 147.

Baker, G. (2003): The Voices of Wittgenstein: The Vienna Circle - Ludwig Wittgenstein and Friedrich Waismann. London: Routledge \& Kegan Paul.

Barwise, J. and Perry, J. (1983): Situations and Attitudes. Cambridge (MA): MIT Press.

Berto, F. (2018): “Aboutness in Imagination.” In: Philosophical Studies. Vol. 175, 1871 - 1886. Berto, F. (2018): “Simple Hyperintensional Belief Revision.” In: Erkenntnis. 1 - 17.

Black, M. (1964): A Companion to Wittgenstein's Tractatus. Ithaca (NY): Cornell University Press. Carnap, R. (1947): Meaning and Necessity. Chicago, London: University of Chicago Press. Ferguson, T.M. (2014): “A Computational Interpretation of Conceptivism.” In: Journal of Applied Non-Classical Logics. No. 4, Vol. 24, $333-367$.

Fine, K. (1986): “Analytic Implication.” In: Notre Dame Journal of Formal Logic. Vol. 27, 169 - 179. Fine, K. (2014): “Truthmaker Semantics for Intuitionistic Logic.” In: Journal of Philosophical Logic.

Vol. 43, $549-577$.

Fine, K. (2015): “Angellic Content.” In: Journal of Philosophical Logic. Vol. 45, 199 - 226.

Fogelin, R.J. (1976): Wittgenstein. London: Routledge \& Kegan Paul.

5 I am very grateful to Lello Frascolla, Peter Hawke, Levin Hornischer, Diego Marconi, Antonio Negro, Naomi Osorio, Aybüke Ozgün, Matteo Plebani, for lovely conversations and useful comments on the subject matter of this paper. 
Frascolla, P. (2007): Understanding Wittgenstein's Tractatus. New York: Routledge.

Gioulatou, I. (2016): Hyperintensionality. ILLC, Universiteit van Amsterdam.

Goodman, N. (1961): “About.” In: Mind. Vol. 70, 1 - 24.

Hawke, P. (2017): “Theories of Aboutness." In: Australasian Journal of Philosophy. Vol. 96, 697 $-723$.

Humberstone, L. (2000): “Parts and Partitions.” In: Theoria. Vol. 66, 41 - 82.

Lewis, D. (1986): On the Plurality of Worlds. Oxford: Blackwell.

Lewis, D. (1988): “Relevant Implication.” In: Theoria. Vol. 54, 161 - 174.

Negro, A. (2017): “Making Sense of Sense Containment." In: History and Philosophy of Logic. Vol. $38,1-22$.

Osorio-Kupferblum, N. (2016): “Aboutness: Critical Notice.” In: Analysis. Vol. 76, 528 - 546.

Parry, W.T. (1933): "Ein Axiomensystem für eine neue Art von Implikation (Analytische Implikation)." In: Ergebnisse eines Mathematischen Kolloquiums. Vol. 4, 5-6.

Perry, J. (1989): “Possible Worlds and Subject Matter." In: Possible Worlds in Humanities, Arts and Sciences. Sture, Allén (ed.). Berlin: De Gruyter, 173 - 191.

Plebani, M. and Spolaore, G. (2018): "Subject Matter: A Modest Proposal." In: Unpublished Manuscript.

Ramsey, F.P. (1923): “Critical Notice.” In: Mind. Vol. 32, 465 - 478.

Ripley, D. (2012): "Structures and Circumstances: Two Ways to Fine-Grain Propositions." In: Synthese. Vol. 189, $97-118$.

Rumfitt, I. (2008): “Knowledge by Deduction.” In: Grazer Philosophische Studien. Vol. 77, 61 84.

Ryle, G. (1933): “About.” In: Analysis. Vol. 1, 10 - 12.

Stenius, E. (1960): Wittgenstein's Tractatus: A Critical Exposition of its Main Lines of Thought. Oxford: Blackwell.

Van Fraassen, B. (1969): "Facts and Tautological Entailments." In: Journal of Philosophy. Vol. 66, $477-487$.

White, R.M. (2006): Wittgenstein's Tractatus Logico-Philosophicus: A Reader's Guide. London: Continuum.

Wittgenstein, L. (1922): Tractatus logico-philosophicus. Ogden, C.K. (trans.), London: Routledge \& Kegan Paul.

Yablo, S. (2014): Aboutness. Princeton, Oxford: Princeton University Press. 